\title{
Leveraging Social Networks To Motivate Individuals to Reduce their Ecological Footprints
}

\author{
Jennifer Mankoff ${ }^{1}$, Deanna Matthews ${ }^{2}$, Susan R. Fussel1 ${ }^{1}$, and Michael Johnson ${ }^{3}$ \\ ${ }^{1}$ HCI Institute, ${ }^{2}$ Department of Civil and Environmental Engineering and \\ ${ }^{3}$ Heinz School of Public Policy \& Management \\ Carnegie Mellon University
}

\begin{abstract}
What role can social networking websites play in supporting large-scale group action and change? We are proposing to explore their use in supporting individual reduction in personal energy consumption. Here we summarize some existing uses of social networking on the web and propose an approach that integrates feedback about ecological footprint data into existing social networking sites and Internet portal sites. Integrating such feedback into popular, commonly used sites allows frequent feedback about performance, while enabling the exploration motivational schemes that leverage group membership. We propose to compare different motivational schemes in three ways: Reduction in $\mathrm{CO} 2$ emission; lifestyle changes; and ongoing use by users who join the site (retention).
\end{abstract}

\section{Introduction}

Americans consumed 100 quadrillion BTUs of energy in 2005 [24]. The residential and commercial sectors of the economy (e.g. households, schools, offices, retail facilities) consumed $40 \%$ of this energy, while industry and transportation consumed $30 \%$ each. Of the energy consumed in homes and businesses, $85 \%$ was generated from fossil fuels such as coal, natural gas, and petroleum. As a result of the combustion of these fossil fuels, residential and commercial energy consumptions created 2.2 billion metric tons of carbon dioxide (CO2), a greenhouse gas presumed to be the major cause of climate change ${ }^{1}$. Seventy three percent of carbon dioxide emissions can be attributed to electric power generation. Everyday activities, such as showering, commuting to work and watching TV result in energy consumption, and thus $\mathrm{CO} 2$ production. This is compounded by energy consumption to provide our lifestyle - to supply water to a home, to deliver gasoline to a gas station, to manufacture the television.

\footnotetext{
1 Throughout this paper, $\mathrm{CO} 2$ emissions are used as a proxy to measure the ecological impact of energy consumption.
}

Murphy argues that "nearly half of Americans' total average ... consumption is more or less under their personal control" [15]. Thus, a solution to reducing greenhouse gas production and the corresponding impact on climate change depends in part on change at the individual level to reduce energy consumption.

Individual choice to reduce energy consumption can already be seen in the increasing popularity and success of local farmers markets, hybrid vehicles, and energy-efficient appliances. These changes have come about as a result of personal decisions rather than mandated requirements. In contrast, legislation to curb energy consumption often focuses on a top-down approach to change. For example, the proposed McCain-Lieberman Climate Stewardship Act mandates reduction of emissions by entities producing more than 10,000 tons of greenhouse gases annually, and completely exempts households.

One barrier to changing consumption habits is educating general citizenry on the links between everyday actions, such as driving to work or leaving a light on while away from home, the energy consumption and emissions generated from those actions, and the resulting ecological impact. Most people have serious misconceptions about climate change [6,7], such as "the hole in the ozone layer is leading to changes in the world's climate" [6], and do not connect climate change to personal energy consumption activities. With knowledge of the link from personal action to ecological impact, people can make better decisions about their actions to minimize their burden on the environment. However, contextual factors such as individual opportunities and abilities may have a big influence [20], pointing at the need for personalized information.

The Internet can play a unique role in increasing individuals' understanding of these links and providing ongoing support for change. In particular, social networking, facilitated by Internet technologies, is a popular and potentially powerful medium for educating consumers and motivating change. We hypothesize that virtual social networks membership can be used to motivate personal change, by enhancing actionable 


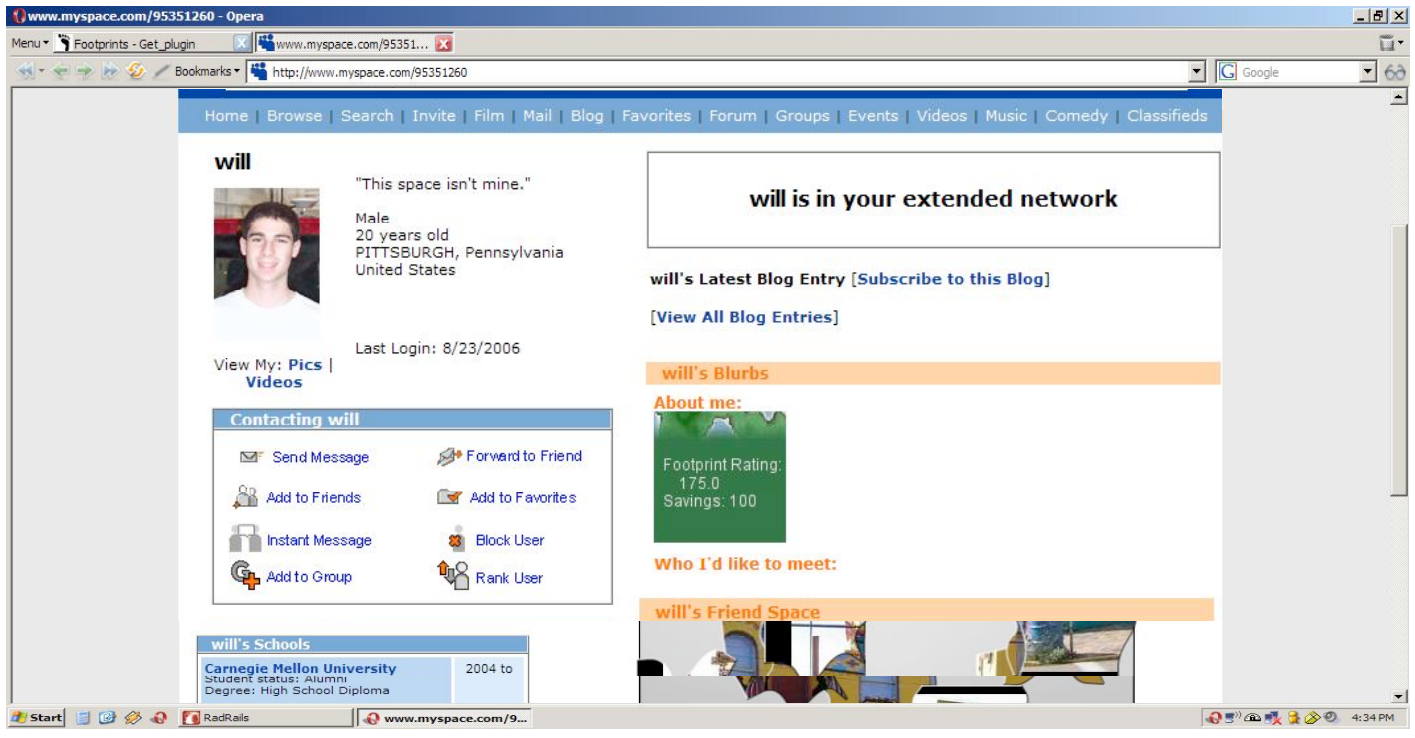

Figure 1: Myspace site modified to show mocked-up CO2 impact in green, middle box under "About me:"

suggestions presented to consumers frequently in an integrated fashion. Our proposal is to piggyback on sites that have tens of millions of members and/or are among the top sites in terms of page views per day. This will allow us to give frequent feedback, and to leverage information about group membership to motivate change. Both frequent feedback and group membership are important sources of motivation.

In the next section, we summarize existing approaches, which include custom, climate-specific social networking sites, and information dissemination about climate change on popular Internet portal sites. We then propose to integrate these approaches by providing personal, customized climate information on popular, generalist sites.

\section{Social Networking Sites}

The Internet currently has $73 \%$ penetration in the American populace [19]. Social networking sites include a large number of those users (for example, there are over 50 million people with MySpace accounts, and in April of 2006 alone there were 38.4 million unique visits to the site [3]). Those people who use the Internet tend to be younger and more affluent than the average American [19]. However, there is also extremely high penetration among middle class families (86\% in 2006 for incomes of $\$ 50-75,000,91 \%$ above that). Likewise, these households tend to have higher energy consumption levels. Households with annual incomes of more than $\$ 50,000$ consumed $40 \%$ $70 \%$ more energy per household than households with annual incomes less than $\$ 30,000$ [11]. These households, while they may have newer and more energy efficient appliances, have more appliances (additional refrigerators and air conditioning), which consume energy.

Thus, the Internet in general, and social networking sites in particular, provide access to a population that includes middle aged professionals (through Yahoo! 360), young adults (through Friendster and MySpace), and a broad range of families (through MySpace, which includes many of the $87 \%$ of teens who are on the internet [13]). An analysis of the top web sites by Nielsen/ NetRatings (http://www.nielsennetratings.com) shows that social networking sites reach over 45\% of web users in April 2006, and among these MySpace is number one. While data is not available on the use of Yahoo! 360, Yahoo! has the "largest unique audience [of home and work Internet users]" of any website [ibid.]

Social networking sites have only recently become a prominent at the scale just described. However, some important studies shed light on how these larger social networking sites are being used. In the past few years, several studies have examined specific social networking sites, including both smaller or currently less popular sites such as Club Nexus [1] and Friendster [8] and more popular sites such as MySpace [18] and Facebook [10].

Unsurprisingly, social networking site members tend to associate with others who have similar values and interests [1]. These personality traits are most often represented through profiles. On many sites, profiles may include collaborative components (such as 
"testimonials" by friends on Friendster [8] and "comments" between friends on MySpace [18]). One important observation that must be addressed when trying to encourage a person to add a new "value" such as footprint reduction to his or her profile is that these sites do not support the sort of multiple "facets" of personality that are so fluidly managed in the real world [8]: Every visitor to a person's profile page, whether friend, family or stranger, sees the same "face," or profile on that page.

Not only is that profile the only face that is present, but doing things that negatively impact that face can have real consequences, for example reducing a person's social capital. A study of Facebook showed that intensive use of the site was a significant predictor of social capital measures such as bonding and bridging [10]. If being ecologically sensitive is perceived to negatively affect social capital, it will likely never spread in such an environment. More plausibly, our proposed work will spread among groups of like-minded folks in what have been termed "echo chambers," but will face much larger hurdles in broad adoption.

\subsection{Presence of the environmental movement on social networking sites}

Although sites such as MySpace and Yahoo! 360 are content-agnostic, MySpace in particular has members who represent organizations, music bands, magazines, and so on. Some environmental-specific MySpace members (organizations, magazines, movies, and so on) include Grist magazine; An Inconvenient Truth; Global Warming: Undo it!; Green-e; Clean Energy; Peak Oil; Native Energy; My Planet; Clean Air Council; Environmental Career Center. MySpace also includes thousands of forums including many on environmentally-relevant politics and science.

Awareness of these MySpace members can spread easily through the social network. As they become "friends" of other members, anyone who visits that other member may encounter them. To add visibility, they may post information about themselves on a "friend's" page. Additional mechanisms such as advertising are available.

These environmental members are somewhat limited, however. They are generally used as ways of disseminating information, and do not provide anything specific to the user or the user's social network. They encourage involvement using a fairly traditional model, in which users visit their sites, see information, and are moved to act.

\subsection{Presence of social networking on environmental sites}

In addition to the presence of environmental and ecological information on social networking sites, social networking methods are beginning to appear on environmental sites. For example, stopglobalwarming.org (http://stopglobalwarming.org), which has over 450,000 members, allows participants to find other members in their zip code. The New American Dream (http://www.newdream.org/) is a social network that encourages members to "live consciously, buy wisely, and join with others in the New Dream Community trying to make a difference." Participant on both sites have "profile" pages that include some personal information. Both sites show participants fresh new suggestions for individual actions/lifestyle changes that can reduce emissions at each visit.

There are also a plethora of sites that do not currently include social networking. Consumers can explore a variety of information sites as well, from numerous "calculators" of ecological impact to web sites such as treehugger (http://treehugger.com/, "the definitive modern+green lifestyle filter") and Terapass (http://terrapass.com/), where individuals can "pay off" their $\mathrm{CO} 2$ emissions.

However, the reach of these sites is limited to those who already have environmental concerns, are likely aware of their burdens, and are likely have already made lifestyle changes to reduce their personal impact.

\subsection{Motivating change}

Societal scale changes have both succeeded and failed many times in the past. Studies of such social movements are an important source of information for our work. Social movement theory has been an active field of study for at least the last quarter century. A recent article by Morris [14] surveys this research broadly, covering a broad range of theoretical stances, but focuses most on the political process model formulated by people such as McAdam, Tarrow, and Tilly. As Morris says, "political process theorists demonstrate that movement mobilization occurs through informal networks, pre-existing institutional structures, and formal organizations... The centrality of mobilizing structures [such as churches] is crucial because it is through them that rational actors figure prominently in the origins of movement." After reviewing the theory, Morris raises some criticisms, and suggests that issues such as leaders, frame lifting and tactics should receive more attention. 
Benford and Snow's article on framing and social movements defines collective action frames as "actionoriented sets of beliefs and meanings that inspire and legitimize the activities and campaigns of a social movement organization" ([5], p. 614). Can framing theory be used to guide intentional social change? William's article on the connection between beliefs and collective action also sheds light on how change might be motivated [25]. He argues that not only do structural opportunities motivate action, but so does culture. For example, he asks if "religious ideological antecedents [are] factors in the emergence of African American social protest."

In trying to explain how and why individuals participate in social movements, Passy and Giugni conducted interviews with activists who did and did not keep a strong commitment to a particular organization (of the Swiss solidarity movement) [16][17]. They found that for strong participation in the movement to be sustained, it was critical that participation be integrated into other life goals and activities of participants ("the interviews show the importance of a sense of coherence and of a holistic view of one's personal life for keeping commitment over time"). They also conducted broad survey-based research of members of the same activist organization. Their second study examines empirically how social networks of different types affect the intensity of individual involvement in a movement. Intensity is defined as giving money, giving occasional time, or giving regular time. They found that networks have three important functions: "structurally connecting prospective participants to an opportunity to participate, socializing them to a protest issue, and shaping their decision to become involved." This represents an alternate view of how best to leverage online social networks.

An example of leveraging theory and knowledge of motivation in group contexts in applied settings is Cosley's article on building member-maintained communities [9]. While we are not focusing on member-maintained communities (such as wikis), the theory may be relevant to using groups to increase motivation in our project.

\subsection{Summary}

Social networking sites can reach a broad range of people who consume energy. But social network participants not already engaged in energy conservation may be reluctant to feature this sort of information on publicly viewable profile pages. At the same time, there are many people already publicly connecting to environmental issues on their profile pages.

Social change is supported in part by mobilizing structures that help to support prominent figures and/or people. Another key facet of social movements is changes in the belief of individuals and the culture they are part of. Strong participation in social movements is most likely when activities can be easily integrated into daily life. This points to the value of personalizing participation to make suggestions more relevant and easier to act on for each individual participant, and of leveraging websites that people already visit frequently for other purposes. Additionally, social networks can support participation both by structurally connecting participants to opportunities and by socializing participants to the issues and shaping their beliefs.

This suggests that social networking sites may be able to play a role in helping to increase individual participation in a social movement and in supporting change, both structurally and by shaping beliefs and culture. However, it is crucial that, to the extent that they suggest concrete changes in energy consumption, these be either diverse enough or personalized enough that some of them can be integrated into existing activities.

\section{Use of social networking channels for providing substantive, action-oriented information}

An interesting opportunity is to directly integrate the personalized data and dynamic suggestions of environmental sites into social networking sites that are frequently visited by people who may not have already bought into the need for change. By using existing sites, we can leverage their structural role as a penetrating component of Internet web use. A complete solution should also consider non-social structures (such as portal pages) to deal with users not yet comfortable displaying their footprint publicly. Finally, we believe that freshness (frequent, new information delivered to the consumer) and personalization (information that is customized based on information about the consumer) may help to address issues of integration mentioned above.

We are proposing to create an information display that can piggyback on large social networking sites such as MySpace (http://www.myspace.com) and Yahoo! 360 (http://360.yahoo.com) and on portal pages such as Google (http://www.google.com/ig?hlen) and will show users information about how well they and the people in their social network are reducing their ecological footprint (See Figure 1 for a mock-up). Our goal is to integrate information on energy 


\begin{tabular}{|c|c|c|}
\hline Simple presentation & Personalized presentation & Values based presentation \\
\hline $\begin{array}{l}\text { Order online instead of driving to } \\
\text { the store }\end{array}$ & $\begin{array}{l}\text { Add CDs to your collection at } \\
\text { CDStore.com }\end{array}$ & $\begin{array}{l}\text { Ordering online instead of driving } \\
\text { long distances alone can save } \\
\text { money; reduce transportation } \\
\text { energy use and increase air quality }\end{array}$ \\
\hline Turn off appliances at the outlet & $\begin{array}{l}\text { Turn off your DVD player and } \\
\text { Stereo at the outlet when not in use }\end{array}$ & $\begin{array}{l}\text { Your appliances my use power even } \\
\text { when "off." Turning them off at the } \\
\text { outlet saves the average American } \\
\$ 150 / \text { year and stops the unnecessary } \\
\text { waste of non-renewable resources }\end{array}$ \\
\hline $\begin{array}{l}\text { Use stand-by mode instead of a } \\
\text { screensaver }\end{array}$ & $\begin{array}{l}\text { Use your laptop's "Better Energy } \\
\text { Savings" mode }\end{array}$ & $\begin{array}{l}\text { Your screensaver uses more power } \\
\text { that most applications. Save battery } \\
\text { time and reduce waste by using } \\
\text { stand-by mode. }\end{array}$ \\
\hline
\end{tabular}

Table 1: Different ways of presenting energy reduction actions may affect adoption.

consumption, energy efficiency, and the links to environmental impacts as seamlessly as possible with websites already in use by millions of people.

These existing social network websites would be enhanced with information about an individual's current environmental footprint savings and goals, the success of their friends, and so on. Users will be shown suggestions for reducing their footprints, but also be encouraged to report creative new ideas. This will help to ensure that fresh material (popular new ideas) is always present on a user's page, helping to draw the user's attention. Users will be able to vote on ideas, and we hope that the new idea system will also encourage participation through competition. Ideas that receive many votes will be analyzed for ecological impact, included as part of an individual's total, and advertised to other users. Thus an idea such as mailing a tree to someone with a large yard, or creating a website to enable carpooling to one's workplace, might get visibility even if they were not part of the original set of suggested actions.

By leveraging popular sites that are visited frequently, we can ensure frequent feedback about changes. Frequent feedback is a critical component of lifestyle change because it can encourage reflection and support informed action. For example, Seligman and Darley's study of feedback about energy consumption showed that a group receiving weekly human-generated feedback about their energy consumption reduced their consumption by $10.5 \%$ compared to a control group after 1 month's use [21]. In another study, Annesi showed that providing computerized feedback about physical activity can increase adherence to an activity goal beyond the typical 6 month drop-out timeframe [1]. However, feedback is most helpful when combined with goal setting. This has been demonstrated in multiple studies of consumer energy conservation [4] [12].
It should be noted that personal values/attitudes and sociodemographic characteristics affect climate change in different ways. In particular, Poortinga, Steg and Vlek found that differences in values were correlated with differences in "intent-oriented" measures of environmental behavior, but actual energy reduction ("impact-oriented" measures) was more strongly correlated with sociodemographic characteristics [20]. One question this leaves open is which sociodemographic communities are most open to making impact-oriented changes when presented with information that may influence their attitudes and values.

Social networking sites also have the advantage of giving us access to groups of people. Group membership can also serve as an education pathway. Competition between groups can improve performance. For example, Siero et al. found that the addition of feedback about a different group's performance increased conservation behavior [23]. Similarly, coordination between groups can help to spread ideas. Simply being part of a group can improve performance (e.g., see Shaw's review of group dynamics [22], and specifically his comments on individual versus group performance).

\subsection{Developing Decision Alternatives for Energy Use}

Given the goals just listed, one problem we must address is how to present energy consumption alternatives to participants to encourage different decision-making and behavior. How do consumers make energy-related decisions? What leads them to make sustainable or unsustainable choices? And how can we present alternatives in the most motivating, informative way possible? As an example, consider the 
action items shown in Table 1. Each column shows the suggests the same action but emphasizes different aspects of that action to engage the reader. How do we know which is best? Some basic questions that we need to answer include:

(a) How can we help participants to understand the impact of their choices on their own lives and on the environment? Are there particular attributes that we should visualize that can help participants to distinguish between alternative choices?

(b) What sort of options do we give participants for "compliance"? For example, is the choice to take action binary or are the gradations of actions?

(c) When looking through multiple options for reducing energy consumption, what sort of decisionmaking process should we support? What attributes of energy consumption are most important to participants? Should we create multi-criteria decision models or should we let users make the choice using their own implicit decision process?

\subsection{Scenarios}

First, consider Sara, an upwardly mobile, middle class, single professional who uses Yahoo! 360 to maintain a social network of friends and business contacts, and uses Google as her home page. She represents an important population because the income and per-capita $\mathrm{CO} 2$ emissions of her peer group is high. She visits the Yahoo! 360 page of a friend, and sees information regarding the current $\mathrm{CO} 2$ emissions of his activities and recent changes he has made to reduce those emissions. She sees that her friend has dropped his net $\mathrm{CO} 2$ emissions by half, and has in the process saved over $\$ 2000$ (via fuel savings, utility savings, etc.). Intrigued, she presses the "More information" link on his page and joins our educational site (called "Footprints"). She is asked for some basic facts (such as her commute length and distance, the make, age and model of her car, and the number of rooms in her home), her Yahoo! 360 account information, and is given instructions for how to update her Yahoo! 360 pages to dynamically display information about "Footprints" each time they are visited. Each time she logs in she sees her current footprint and dollars saved and suggestions for simple changes she can make (like replacing a light bulb with a fluorescent bulb, or buying an Energy Star ${ }^{\circledR}$ labeled appliance). She also sees how much money she has saved by making these changes, and she sees highlights of great ideas by other users that have received many positive votes from "Footprints" members. One day, she sees that a new rideshare program in her zip code has gotten many votes.
Intrigued, she clicks on it, and discovers that other members of her network have joined. She could shorten her commute, save money, and lower her footprint by joining as well. She adds herself to the rideshare group and signs up to carpool once a week to try it out ....

Second, consider Sammy. Sammy is a middle school student who has been a member of Footprints for some time now, along with MySpace (where he originally found out about Footprints when one of his friends posted the information on his MySpace page). While Sammy himself does not make major decisions about energy usage, he is more liberal than his parents, as are many of his peers. He represents a population who (1) will be making important decisions affecting $\mathrm{CO} 2$ emissions and policy in the future and is thus important to educate and (2) can influence a group of people (the parents) who might normally not change their lifestyle to reduce $\mathrm{CO} 2$ emissions. Sammy is part of the school chess club, and they have convinced their parents and the school administration that each dollar they save by reducing the ecological footprint of their homes and school should be donated to the club. Their goal is to attend the state tournament in three months. Sammy and his club have already done all the "obvious" things they can, such as convincing their parents to turn down the thermostat at home, and taking the bus to the mall, rather than asking for a ride, and they have raised almost half of the money they need this way. However, they'd like to raise more money more quickly, so they are also exploring creative ideas for change. Their current plan is to convince the school to let them start a garden to provide some of the vegetables used in the school lunch program. When they post this idea on the Footprints site it gets lots of votes from clubs at other schools, prompting similar programs around the country. As a result of this, those schools start an interschool competition to come up with the best idea for lowering the entire school footprint....

Naturally, both of these scenarios represent idealized views of how our system might work. For example, it's not clear from the scenarios if Sara and Sammy understanding (a) how much change in their energy behavior they will undergo if they choose specific alternatives, (b) the impact on aggregate energy usage of their changes (or the total impact, positive or negative, of many users making the same choice as they do) and (c) tradeoffs between different energy reduction alternatives with respect to personal inconvenience, cost and attitudes. What are the real-life constraints that might affect their choice to accept, not accept, or partially act on a suggested change? 


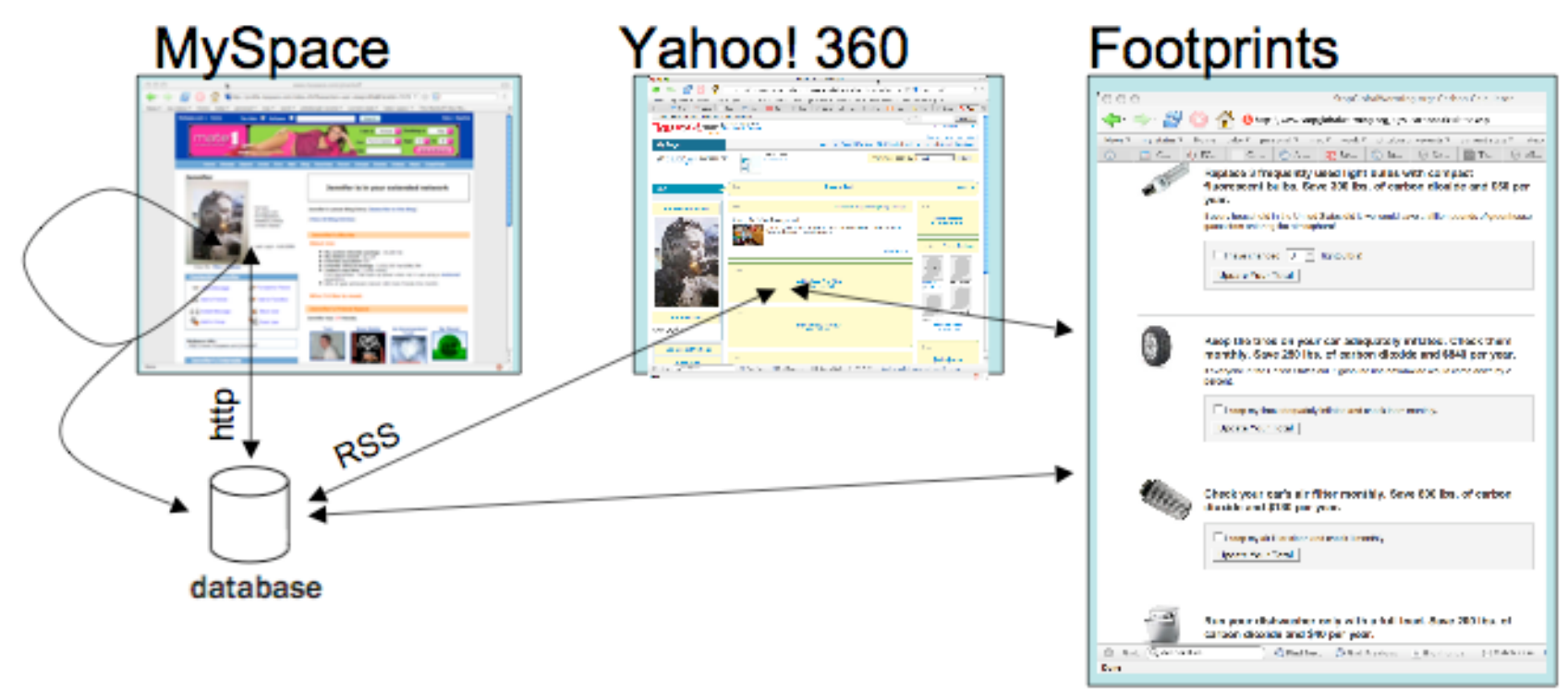

Figure 3: Architecture diagram for the Footprints system. Images are mock-ups. Users will typically view and update information through their social networking pages. Where Web 2.0 technologies such as Javascript are supported, all interaction will take place without leaving the social networking site (MySpace is an example of this). For less sophisticated sites, we will export RSS data to show status and feedback to the user. In this case, interaction will be done by clicking on a link that takes the user to a custom "Footprints" site where his or her task can be completed, causing an update to the database and sending the user back to his or her original social networking page.

\section{Implementation}

The proposed system's technological implementation will focus on usability, transparency, and integration with existing sites. Our development specifically targets MySpace and Yahoo! 360, and also includes support for other frequently visited sites.

Figure 3 illustrates our planned system architecture. We can display dynamic suggestions for energy use reduction on many sites without direct access by leveraging Web 2.0 technologies, such as Javascript, GreaseMonkey, RSS, and so on. Web 2.0 technologies make it possible to treat the web as a platform for developing applications, rather than an information repository. They make it possible to add dynamic, interactive data to the web. The options we plan to support include:

- Secure RSS feeds that can be added to any portal supporting RSS (including Yahoo!)

- "Badges" - dynamically generated images, such as the green box under "about me" in Figure 1, that can be added to pages such as MySpace

- A Google "module" (a custom minicomponent that can be added to an individual's Google portal page)

- A GreaseMonkey module that can allow the user to add the Footprints display to any arbitrary website (typically this would be one that they visit often)

- A Webservices API for those who wish to create custom forms of access

- A cellphone module for mobile access

Once a participant views a suggested change using one of the methods just described, he or she will be able to click on a link or menu associated with the suggested action to report on what change he plans to make. We will then follow up by asking participants if they have taken the action just described. In addition to self-reporting, we plan to allow sensor integration. Possible sensors might include a device that can tell whether a user is walking or driving, or a software sensor that tracks occurrences of online purchases.

We will develop a database back-end and web pages needed to create an account, enter footprint data, view and update one's footprint, and view the footprint of others whom a user has recruited. The back-end database will include information about number of people recruited, friends, current footprint, and changes made (such as changing the thermostat temperature). It will also include a table of actions used to generate suggestions to present to users.

In addition to basic functionality, we will add support for presenting dynamic suggestions about changes to users, and expand our system to support groups. A user will be able to access information about: people within a certain number of miles of his 
or her zip code; people that the user has recruited; and people in a user's social network. Users will be able to compare the performance of these groups to other similar groups. To support this, we will add group membership information to the database, and update the security measures to support appropriate limits on access to group member pages. We will enhance the web pages with group-related functionality. Also, we will add information about group performance to the RSS, Javascript and PHP modules.

While we can present users with common solutions to reducing ecological footprint, one of the advantages of having a large network of users is that they can generate creative ideas for change. We will support reporting and voting on creative ideas. Each user will be able to report on creative ideas and put them up for votes by other users. Our software will randomly share new ideas among users, who can vote on them. Ideas that accumulate many votes will be shown more frequently. In addition to top-rated ideas, user's modules will show the top idea in their zip code or social network.

\section{Deployment plan}

Once we have worked out any usability problems with the software, we will begin phasing in large-scale deployments. Our initial roll-out plan is to select a well-defined community, such as a small college campus, and attempt to reach critical mass within that community. Our first step will be to give talks and advertise on a specific college campus and work to get a high percentage of students to join.

The goal of this deployment will be to explore the value of groups. We will start by gathering baseline data on the community as it begins to use our software. Once we have established ourselves, we will begin testing the value of dynamic suggestions about changes. Later in the year, after support for groups is completed, we will begin exploring the impact of intergroup competition.

A major focus of this deployment will be to explore different motivational approaches, including presenting individual goals, group goals, leader boards, and so on. We will test three strategies:

Individual goals: We will provide users with daily information and suggestions for how to make changes. Users can self-report which advice they followed, which will update their footprint and dollars saved.

Goal setting: Goal setting, both individual, within group, and between groups, can be successful at encouraging change, particularly in combination with frequent feedback.
Competition: We will explore the value of competition in encouraging change. For example, we expect users to compete to reach the top of leader boards. Competition can be used positively to encourage recruitment of new users and to encourage reaching goals. Because changes are self-reported, competition will focus on things that are verifiable to reduce trust. In particular, we will allow competition for number of other members recruited. We will reward successful recruiters.

We will conduct a four-month long comparative study of the techniques just described. Our baseline data will show the value of simply showing information in a fresh way (strategy S1). We will compare this to setting individual goals (S2), setting group goals (S3), and competition (S4). We will divide the members of the campus into subgroups based on their social networks. We will assign a different strategy (S1-S4) to each sub-group, in such a way that sub-groups are balanced for their baseline performance. We will end with another baseline period. Additionally, we will interview participants after the study is complete to learn more about what led them to decide to take or not to take specific actions that were suggested to them. Our hypothesis is that competition will perform best, followed by setting group goals, then setting individual goals, and then simply showing information. This is supported by prior work, which shows that membership in groups, and feedback about the performance of different groups can increase conservation behavior [23][22].

Concurrently with this study, we plan to begin a larger-scale deployment, so that we can begin to understand the impact of large-scale participation on the technologies we are developing. We plan to use the following strategies to reach large numbers of users:

First, we will work hard to develop a partnership with existing grassroots ecologically oriented websites that have already developed a large base of users.

Second, we will encourage users to recruit members. By allowing member recruitment to help with goal attainment and by promoting competition around recruitment, we hope to achieve high results.

Third, we will leverage social networking sites. Users of sites such as MySpace and Yahoo! 360 spend time online communicating with friends. Even without direct support from existing websites, we can ensure that our data is visible to anyone who visits a given person's page. Each time one of those friends visits a page featuring our additions, they may choose to join our campaign. Additionally, it is part of the culture on sites such as MySpace to advertise new ideas to friends 
(via "bulletins") and to invite new people to join an initiative.

Fourth, we plan to create simple interfaces for people to "join in" such as one-click download and install or invite buttons to help "spread the word." Over time, we hope that a combination of word-ofmouth, publicity, and direct adoption by commonly frequented websites will make our software accessible to a large cross-section of existing popular socialnetworking sites.

Fifth, we will advertise. Many options for advertisement on the Internet are free. For example, Google provides free advertising to non-profits for limited periods of time. Additionally, MySpace has an existing culture of advertising.

\section{Conclusions}

Today's Internet is a social place, and a place that individuals visit daily. Social networking has an important role to play in supporting education and personal change of energy consumption and emissions. This can be seen in the presence of energy and environmental information on the most widely used social networking site (MySpace), and in the application of social networking principles to sites that focus on the environment. We argue that a combination of the two approaches could provide action-oriented, dynamic, personalized information to consumers on the portal and networking sites that they visit most frequently. This would make it possible to reach a broad segment of the population frequently, and to provide information that would be optimally motivating.

An approach that leverages frequently visited social network sites that include millions of people, could have a powerful impact on individual performance. Frequent viewing will increase the likelihood that users encounter regular reminders and suggestions for change. By leveraging popular websites, we will be able to provide frequent reminders and motivators, as well as feedback about users' relative performance. By piggybacking on websites that consumers already visit frequently, we can guarantee that feedback and goals generated by our system are seen often enough to have an impact, without requiring that the consumer remember to check, installing custom feedback interfaces (which would limit the possible size of our deployment) or annoying the consumer with reminders that come at inappropriate times or seem too frequent. Our proposed approach also leverages information about a users' social network. This will enable us to encourage change on the basis of group membership. We plan to leverage existing knowledge about groups to design an experience that will be optimally effective at motivating users.

This work will help to provide information about Web 2.0 technologies through the process of iteratively designing and deploying them. Additionally, our deployment will generate large-scale field data about approaches for increasing motivation, retention, trust, performance of groups, and about what particular suggested changes are most actionable.

\section{References}

[1] Adamic, L., Buyukkokten, O. and Adar, E. (2003) "A social network caught in the Web." First Monday.

[2] Annesi, J. J. (1998) Effects of computer feedback on adherence to exercise. Perceptual and Motor Skills, 87:723-730.

[3] Bausch, S. and L. Han (2006). Social networking sites grow 47 percent, year over year, reaching 45 percent of web users, according to Nielsen/NetRatings. Nielsen/NetRatings. http://www.netratings.com/pr/pr 060511.pdf

[4] Becker, L. J. (1978) Joint effect of feedback and goal setting on performance: a field study of residential energy conservation. Journal of Applied Psychology, 63(4):428-433, Jan, 1978

[5] Benford, R. D. and Snow, D. A. (2000) Framing processes and social movements: An overview and assessment. Annual Review of Sociology 26:611-39, 2000.

[6] Bostrom, A., Morgan, M. G., Fischoff, B. and Read, D. (1994) What do people know about climate change? 1. Mental Models. Risk Analysis, 14(6):959-969, 1994.

[7] Bostrom, A., Morgan, M. G., Fischoff, B. and Read, D. (1994) What do people know about climate change? 2. Survey studies of educated lay people. Risk Analysis, 14(6):971-982, 1994.

[8] boyd, d. and Heer, J.. (2006). "Profiles as Conversation: Networked Identity Performance on Friendster." Proceedings of the Hawai'i International Conference on System Sciences (HICSS-39), Persistent Conversation Track. Kauai, HI

[9] Cosley, D., (2005) Mining social theory to build member-maintained communities. Proceedings of KCVC 2005, Palo Alto, CA

[10] Ellison, N., Steinfield, C. and Lampe, C. (2006) Spatially Bounded Online Social Networks and Social Capital: The Role of Facebook. Paper presented at the Annual Meeting of the International Communication Association, Dresden, June 19-23.

[11] Energy Information Administration (2001). Residential energy Consumption Survey Consumption and Expenditures Tables http://www.eia.doe.gov/emeu/recs/recs2001/detailcetbls. html Last updated Nov 2004. Accessed 06 June 2006.

[12] van Houwelingen, J. H. and W. F. van Raajj (1989). The Effect of Goal-Setting and Daily Electronic Feedback on In-Home Energy Use. Journal of Consumer Research, 16(1):98-105. 
[13] Lenhart, A., Madden, M. and Hitlin, P. (2005). Teens and Technology: Youth are leading the transition to a fully wired and mobile nation. Pew Internet and the American Life Project, July 27, 2005.

[14] Morris, A. (2000) Reflections on Social Movement Theory: Criticisms and Proposals. Contemporary Sociology, 29(3):445-454. 2000.

[15] Murphy, P. (2006) Post-Peak - The change starts with us. Energy Bulletin. Published Thursday, March 23, 2006. Available at http://www.energybulletin.net/print.php?id=14143

[16] Passy, F. and Giugni, M. (2000) Life-Spheres, Networks and Sustained Participation in Social Movements: A Phenomenological Approach to Political Commitment. Sociological Forum, 15(1):117-144, 2000.

[17] Passy, F. and Giugni, M. (2000) Social Networks and Individual Perceptions: Explaining Differential Participation in Social Movements. Sociological Forum, 16(1):123-153, 2000.

[18] Perkel, D. (2006) Copy and Paste Literacy: Literacy Practices in the Production of a MySpace Profile. Informal Learning and Digital Media. Odense Denmark: September 21-23.
[19] Pew Internet and American Life project (2006) Demographics of Internet Users, April, 2006. http://www.pewinternet.org/trends/User Demo 4.26.06. htm

[20] Poortinga, W., Steg, L. and Vlek, C. (2004) Values, environmental concern, and environmental behavior: A study into household energy use. Environment and Behavior 36(1):70-93.

[21] Seligman, C. and J. M. Darley (1977). Feedback as a means of decreasing residential energy consumption. Journal of Applied Psychology 62(4):363-368.

[22] Shaw, M. E. (1961). Group Dynamics. Annual Review of Psychology, 12:129-156.

[23] Siero, F.W., A. B. Bakker, G. B. Dekker and M. T. C. van Denburg (1996) Changing organizational energy consumption behavior through comparative feedback. Journal of Environmental Psychology, 16(3):235-246.

[24] U.S. Department of Energy (2006) Annual Energy Review 2005. Energy Information Administration, Washington, DC DOE/EIA-0384.

[25] Johnny E. Williams. Linking Beliefs to collective action: politicized religious beliefs and the civil rights movement. Sociological Forum, 17(2):203-222, 2002. 